\title{
New MACRO results on atmospheric neutrino oscillations
}

\author{
G. Giacomelli and A. Margiotta \\ Dipartimento di Fisica dell'Università di Bologna \\ and INFN, Sezione di Bologna, I-40127 Bologna, Italy \\ giacomelli@bo.infn.it, margiotta@bo.infn.it \\ For the MACRO Collaboration* \\ Invited Paper at the NANP03 Int. Conf., Dubna, 2003
}

November 9, 2018

\begin{abstract}
The final results of the MACRO experiment on atmospheric neutrino oscillations are presented and discussed. The data concern different event topologies with average neutrino energies of $\sim 3$ and $\sim 50 \mathrm{GeV}$. Multiple Coulomb Scattering of the high energy muons in absorbers was used to estimate the neutrino energy of each event. The angular distributions, the $L / E_{\nu}$ distribution, the particle ratios and the absolute fluxes all favour $\nu_{\mu} \rightarrow \nu_{\tau}$ oscillations with maximal mixing and $\Delta m^{2}=0.0023 \mathrm{eV}^{2}$. A discussion is made on the Monte Carlos used for the atmospheric neutrino flux. Some results on neutrino astrophysics are also briefly discussed.

Keywords: Astroparticle physics; underground detectors; neutrino oscillations.

PACS Nos.: 13.15.+g; 14.60.Pq; 96.40.De; 96.40.Tv
\end{abstract}

\section{Introduction}

MACRO was a large area multipurpose underground detector [1 designed to search for rare events and rare phenomena in the penetrating cosmic radiation. The experiment obtained important results on atmospheric $\nu_{\mu}$ oscillations and performed $\nu_{\mu}$ astronomy studies, indirect searches for WIMPs and searches for low energy $\bar{\nu}_{e}$ from stellar gravitational collapses [2].

The detector was located in Hall B of the underground Gran Sasso Lab at an average rock overburden of 3700 m.w.e.; it started data taking with part of the apparatus in 1989; it was completed in 1995 and was running in its final configuration until December 2000.

The detector had global dimensions of $76.6 \times 12 \times 9.3 \mathrm{~m}^{3}$ and provided a total acceptance to an isotropic flux of particles of $\sim 10,000 \mathrm{~m}^{2} \mathrm{sr}$. The detector was composed of three horizontal layers of liquid scintillation counters, 14 layers of limited streamer tubes and one layer of nuclear track detectors, Fig. 11 Vertically it was divided into two parts: the lower part contained 10 layers of streamer tubes, 7 layers of rock absorbers and 2 layers of liquid scintillators; the upper part was empty, contained the electronics and was covered by 1 layer of scintillators and 4 layers of streamer tubes. The sides of the detector were covered with 1 layer of scintillators and 6 layers of limited streamer tubes, so as to obtain a closed box structure. Each of the subdetectors could be used in "stand-alone" and in "combined" mode.

*see Ref. [1] for a list of MACRO Authors and Institutions 
In the following we shall briefly recall neutrino oscillation formulae and atmospheric neutrinos; then we shall discuss our main results including the ratio of vertical to horizontal muons and the study of the $L / E_{\nu}$ distribution. After a detailed analysis of the present situation of the different Monte Carlos (MCs), we summarize our atmospheric neutrino oscillation results and we conclude with a short discussion of some neutrino astrophysics studies.

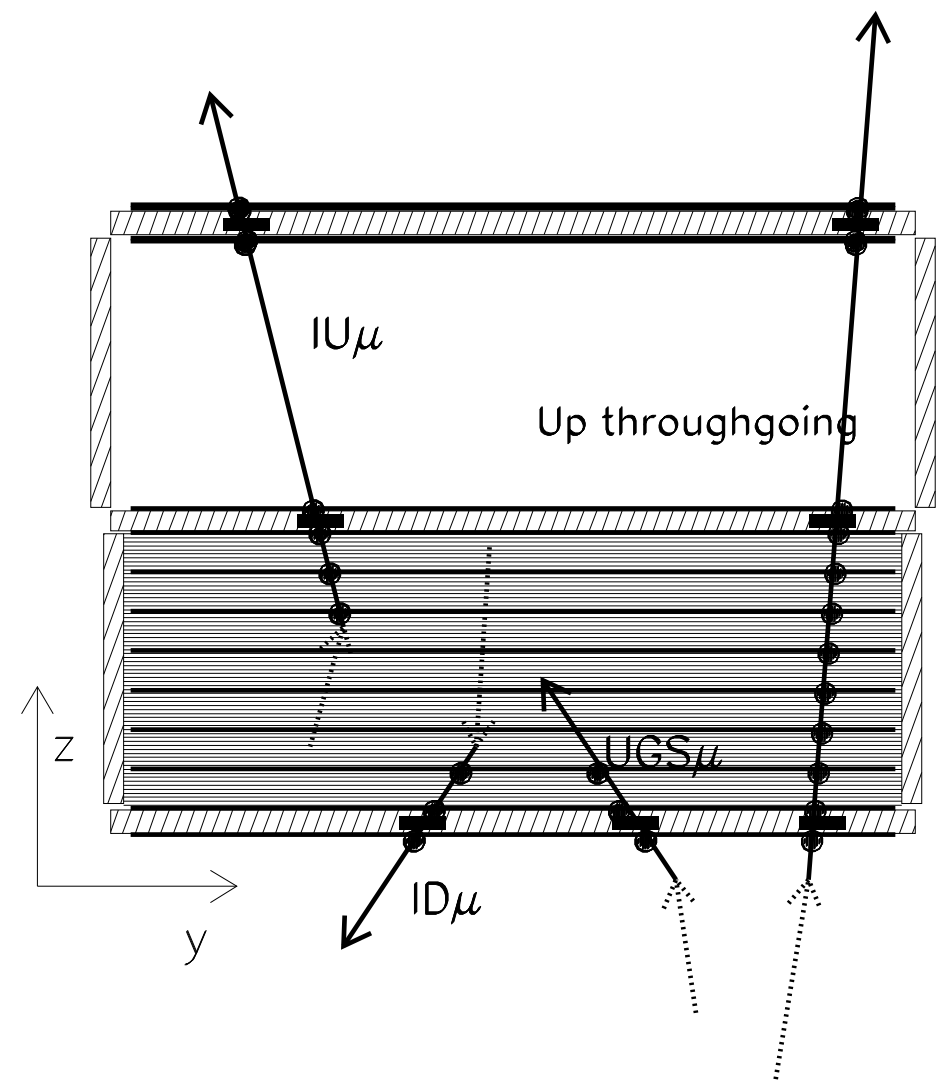

Figure 1: Cross section of the detector, which also shows the various atmospheric neutrino topologies measured. The horizontal scintillators are indicated as dashed boxes, the streamer tubes as horizontal thick black lines, the rock absorbers as boxes with horizontal thin lines. The sides of the detector were formed by 1 layer of scintillators and 6 layers of streamer tubes.

\section{Atmospheric neutrino oscillations. Monte Carlos}

Neutrino oscillations. If neutrinos have non-zero masses, one has to consider the 3 weak flavour eigenstates $\nu_{e}, \nu_{\mu}, \nu_{\tau}$ and the 3 mass eigenstates $\nu_{1}, \nu_{2}, \nu_{3}$. The flavour eigenstates $\nu_{l}$ are linear combinations of the mass eigenstates $\nu_{m}$. Neutrino oscillations depend on six independent parameters: two mass-squared differences, $\Delta m_{12}^{2}$ and $\Delta m_{23}^{2}$, three mixing angles $\theta_{12}, \theta_{13}, \theta_{23}$ and the CP-violating phase $\delta$. In the simple case of two flavour eigenstates $\left(\nu_{\mu}, \nu_{\tau}\right)$ which oscillate with two mass eigenstates $\left(\nu_{2}, \nu_{3}\right)$, and $\delta=0$ one has:

$$
\begin{gathered}
\nu_{\mu}=\nu_{2} \cos \theta_{23}+\nu_{3} \sin \theta_{23} \\
\nu_{\tau}=-\nu_{2} \sin \theta_{23}+\nu_{3} \cos \theta_{23}
\end{gathered}
$$


The survival probability of a $\nu_{\mu}$ beam is

$$
P\left(\nu_{\mu} \rightarrow \nu_{\mu}\right) \simeq 1-\sin ^{2} 2 \theta_{23} \sin ^{2}\left(\frac{1.27 \Delta m_{23}^{2} \cdot L}{E_{\nu}}\right)
$$

where $\Delta m_{23}^{2}=m_{3}^{2}-m_{2}^{2}$ and $\mathrm{L}$ is the distance travelled by the neutrino of energy $E_{\nu}$ from production to detection. The two-neutrino approximation is adequate for discussing our data.

Atmospheric neutrinos. High energy (HE) primary Cosmic Rays (CRs), protons and nuclei, interact in the upper atmosphere producing a large number of pions and kaons, which decay yielding muons and muon neutrinos; then muons decay yielding $\nu_{\mu}$ 's and $\nu_{e}$ 's. The ratios of their numbers are $N_{\nu_{\mu}} / N_{\nu_{e}} \simeq 2$ and $N_{\nu} / N_{\bar{\nu}} \simeq 1$. The atmospheric neutrinos are produced in a spherical shell at about 10-20 km above ground and proceed towards the Earth. Atmospheric neutrinos have energies from a fraction of $\mathrm{GeV}$ up to more than $100 \mathrm{GeV}$ and they travel distances $\mathrm{L}$ from few tens of $\mathrm{km}$ up to $\sim 13000 \mathrm{~km}$. An underground detector is "illuminated" by a flux of neutrinos from all directions and it can make oscillation studies for $1<L / E_{\nu}<10^{4} \mathrm{~km} / \mathrm{GeV}$.

MACRO detected upgoing muon neutrinos via charged current interactions, $\nu_{\mu} \rightarrow \mu$; the upgoing muons were identified with the streamer tube system (for tracking) and the scintillator system (for time-of-flight measurement). The events measured and expected for the three measured topologies, indicated in Fig. 1 and the $L / E_{\nu}$ distribution deviate from Monte Carlo expectations without oscillations, Figs 2 , 4 and Tables 1, 2; the deviations point to the same $\nu_{\mu} \rightarrow \nu_{\tau}$ oscillation scenario [2]-8].

Monte Carlos. The measured data of Fig. 2 were compared with different MC simulations. In the past we used the neutrino flux computed by the Bartol96 group [9] and the GRV94 [10] parton distribution. For the low energy channels the cross sections in [11] were used; the propagation of muons to the detector was done using the energy loss calculation by Lohmann et al. 12. The total systematic uncertainty in the predicted flux of upthroughgoing muons, adding in quadrature the errors, was estimated to be $\pm 17 \%$; this is mainly a scale error that does not change the shape of the angular distribution. The error on the shape of the distribution is $\sim 6 \%$. The detector was simulated using GEANT [13]. A similar MC (Honda96) was used by the Superkamiokande Collaboration [14].

Recently new improved MC predictions were made available by the Honda 14 and Fluka 15] groups. They include three dimensional calculations of hadron production and decays and of neutrino interactions, improved hadronic model and new fits of the primary cosmic ray flux. The two MCs yield predictions for the non oscillated and oscillated $\nu_{\mu}$ fluxes in complete agreement with each other, equal to within few \%, see Figs. 3a, b. The shapes of the angular distributions for oscillated and non oscillated Bartol96, new Fluka and new Honda fluxes are the same to within few $\%$, Fig. 3a, b. The absolute values of our upthroughgoing muon data are about $20-30 \%$ above those predicted by the new Fluka and Honda MCs; this situation is also true for the new Superkamiokande data [16; the measurements of the flux value at $\cos \vartheta=0$ performed by various experiments do not seem to be conclusive [17. Note in Fig. 3a that the new Fluka muon flux with an older cosmic ray fit is considerably above the new Fluka flux with the new CR fit. Our high energy $\nu_{\mu}$ data suggest that the new Honda and Fluka predictions should be raised by about $20-25 \%$, probably because of the used new CR fit. The inclusion of the new ATTIC Collaboration measurements of primary CRs seems to lead to the old energy dependence of $E^{-2.71}[18$. Thus the Bartol96 MC may probably still be used for the prediction of the absolute flux, besides predicting well the shape of the angular distribution. In the following we shall use mainly the predictions of this MC. It should be noted that the evidence for neutrino oscillations rests primarely with the shape of the angular distribution and this is the same in all MC calculations. 


\section{MACRO results on atmospheric neutrinos.}

The upthroughgoing muons come from $\nu_{\mu}$ interactions in the rock below the detector; the $\nu_{\mu}$ 's have a median energy $\bar{E}_{\nu} \sim 50 \mathrm{GeV}$; muons with $E_{\mu}>1 \mathrm{GeV}$ cross the whole detector. The data taking livetime was slightly over 6 years (full detector equivalent). The data, Table 1 and Fig. 2 $\mathrm{a}$, deviate in shape and in absolute value from the Bartol96 MC predictions (see Section 2). This was first pointed by MACRO in 1995 [4].
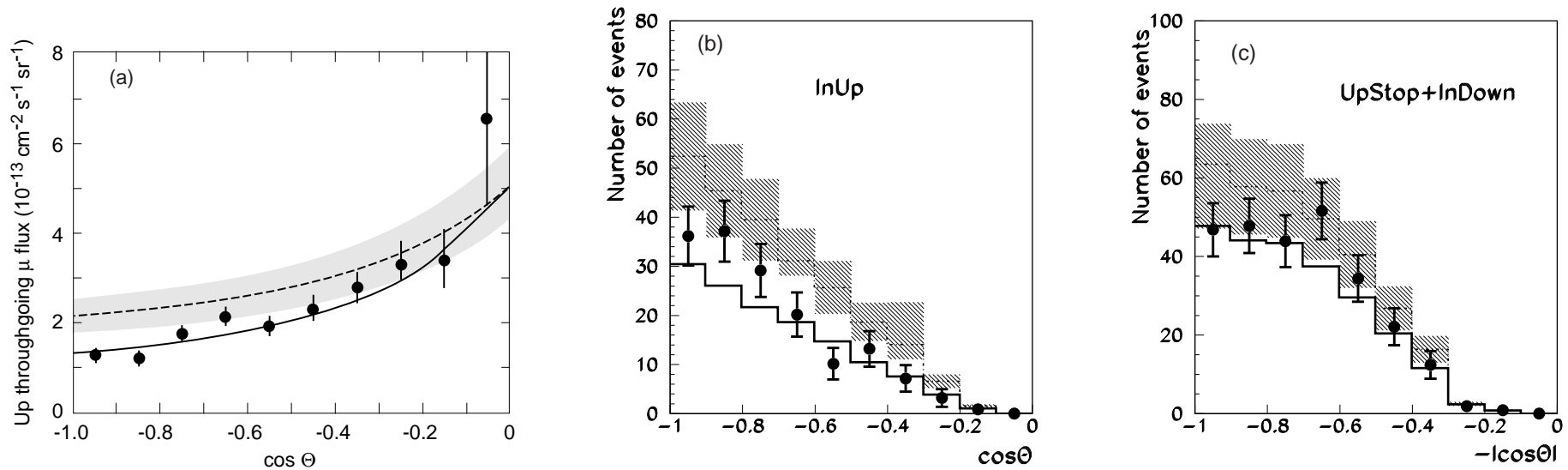

Figure 2: Zenith distributions for the MACRO data (black points) for (a) upthroughgoing, (b) semicontained and (c) up-stopping muons + down semicontained. The dashed lines are the no-oscillation MC predictions Bartol96 in (a) and new FLUKA in (b) and (c) (with scale error bands); the solid lines refer to $\nu_{\mu} \rightarrow \nu_{\tau}$ oscillations with maximal mixing and $\Delta m^{2}=2.3 \cdot 10^{-3} \mathrm{eV}^{2}$.

A large number of possible systematic effects and backgrounds that could affect the measurements were studied. One of the most significant checks was performed using only the scintillator system with two different and independent electronic systems $\underline{3}, 8]$. Two different final analyses of the upthrough data have been performed: they agree to within $5 \%$.

Assuming no oscillations, the number of expected upthroughgoing muon events integrated over all zenith angles from Bartol96 without oscillations is 1169; the measured number is 857, Table 1. Thus the ratio of the observed number of events to the Bartol96 expectation is 0.73.

$\nu_{\mu} \rightarrow \nu_{\tau}$ versus $\nu_{\mu} \rightarrow \nu_{s}$. Matter effects due to the difference between the weak interaction effective potential for muon neutrinos with respect to sterile neutrinos $\left(\nu_{s}\right)$ would produce a different total number and a different zenith angle distribution of upthroughgoing muons. The ratio $R_{1}=$ Vertical/Horizontal $=N(-1<\cos \theta<-0.7) / N(-0.4<\cos \theta<0)$ was used to test the $\nu_{\mu} \rightarrow \nu_{s}$ oscillation hypothesis versus $\nu_{\mu} \rightarrow \nu_{\tau}$ [2], 6], 8]. The measured value is $R_{\text {meas }}=1.38$; it should be compared to $R_{\tau}^{\min }=1.61$ and $R_{\text {sterile }}^{\min }=2.03$, which are the minimum

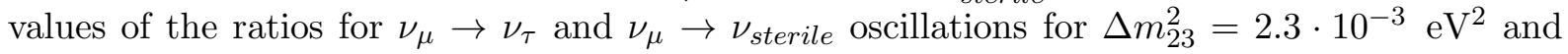
maximal mixing. For $\nu_{\mu} \rightarrow \nu_{\tau}$, the one-sided probability to measure a value lower than $R_{\text {meas }}$ is $7.2 \%$. For $\nu_{\mu} \rightarrow \nu_{s}$ the probability is $P_{\text {ster }}^{\text {best }} \simeq 1.5 \cdot 10^{-4} ; P_{\tau}^{\text {best }}$ is about 480 times larger than $P_{\text {ster }}^{\text {best. }}$. Therefore $\nu_{\mu} \rightarrow \nu_{s}$ oscillations (with any mixing) are excluded at about $99.8 \%$ c.l. with respect to $\nu_{\mu} \rightarrow \nu_{\tau}$ oscillations with maximal mixing [8].

Oscillation probability as a function of the ratio $L / E_{\nu} . E_{\nu}$ was estimated by measuring the muon energy, $E_{\mu}$, by means of the muon Multiple Coulomb Scattering (MCS) in the rock absorbers in the lower MACRO. The best method employed the streamer tubes in "drift mode", using the special electronics designed to search for magnetic monopoles [19]. The 


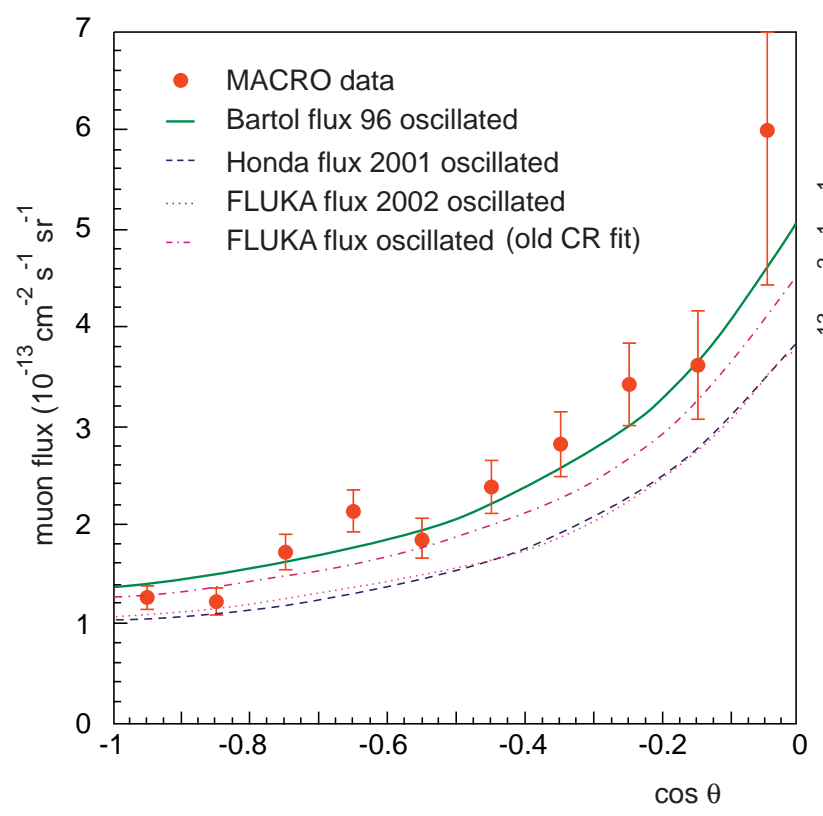

(a)

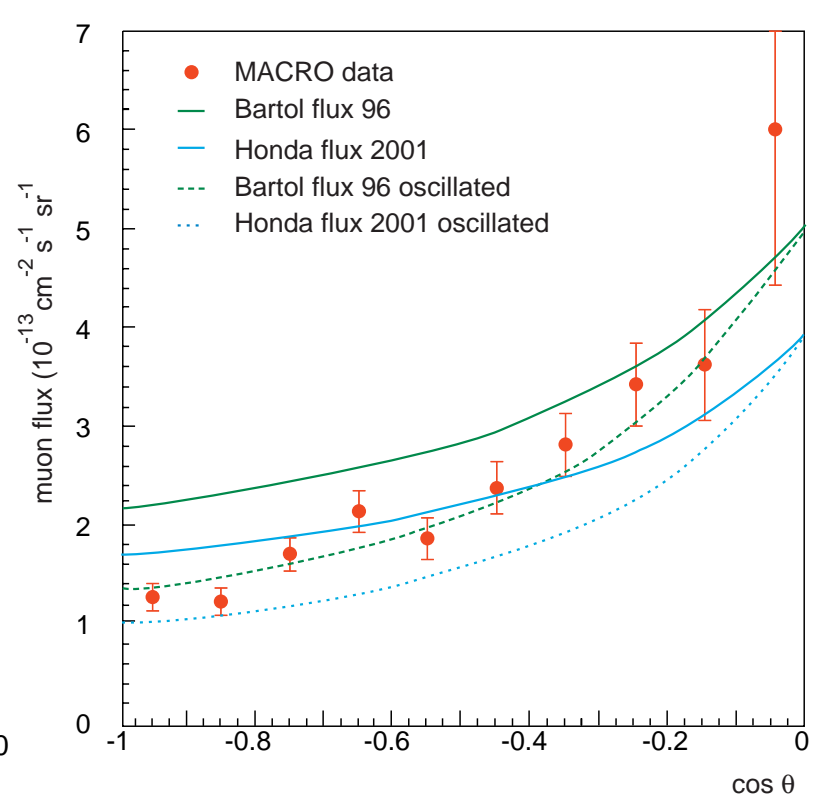

(b)

Figure 3: (a) Comparison of the measured angular distribution for upthroughgoing muons and the oscillated flux obtained from different MCs (for $\Delta m^{2}=2.3 \cdot 10^{-3} \mathrm{eV}^{2}$ and maximal mixing). The solid line is the Bartol96 flux, the dotted and the dashed lines at the bottom are the new Fluka and Honda fluxes; the dotted-dashed line in the middle is the Fluka flux using a different (older) fit to the cosmic ray data. (b) Comparison of our measurements with the Bartol96 and the new Honda 2001 oscillated and non oscillated fluxes.

\begin{tabular}{ccc}
\hline Topology & Measured events & $(\text { Data } / M C)_{\text {noosc }}$ \\
\hline Upthroughgoing muons & 857 & 0.73 \\
Semicontained (UP) & 157 & 0.55 \\
Upstopping + Down & 262 & 0.74 \\
semicontained (ID + UGS) & & \\
\hline
\end{tabular}

Table 1: For each topology the table gives the number of events measured and the ratios $(\text { Data } / M C)_{\text {noosc }}$ using the Bartol96 MC.

space resolution achieved was $\simeq 3 \mathrm{~mm}$. For each muon, seven variables were given in input to a Neural Network (NN) previously trained to estimate muon energies with MC events of known input energy crossing the detector at different zenith angles. The distribution of the ratio $R=\left(\right.$ Data $\left./ M C_{\text {noosc }}\right)$ obtained by this analysis is plotted in Fig. 4 as a function of $\left(L / E_{\nu}\right)$ [7. Notice that the data extend from $\left(L / E_{\nu}\right) \sim 30 \mathrm{~km} / \mathrm{GeV}$ to $5000 \mathrm{~km} / \mathrm{GeV}$.

The Internal Upgoing (IU) muons ( 157 events, 285 expected by Bartol96 and 235 expected by new FLUKA, Table 1) come from $\sim 4 \mathrm{GeV} \nu_{\mu}$ 's interacting in the lower apparatus, Fig. 1. Compared to the no-oscillation prediction there is a reduction of about a factor of two in the flux of these events, without any appreciable distortion in the shape of the zenith distribution, Fig. 2] [5], [8]. The MC predictions for no oscillation in Figs. 2b and 2r are given by the dashed lines with a $21 \%$ systematic scale band. Notice that at these energies, the new Honda and Fluka MC predictions are low by about $12 \%$.

The upstopping muons (UGS) are due to $\sim 3 \mathrm{GeV} \nu_{\mu}$ 's interacting below the detector and 


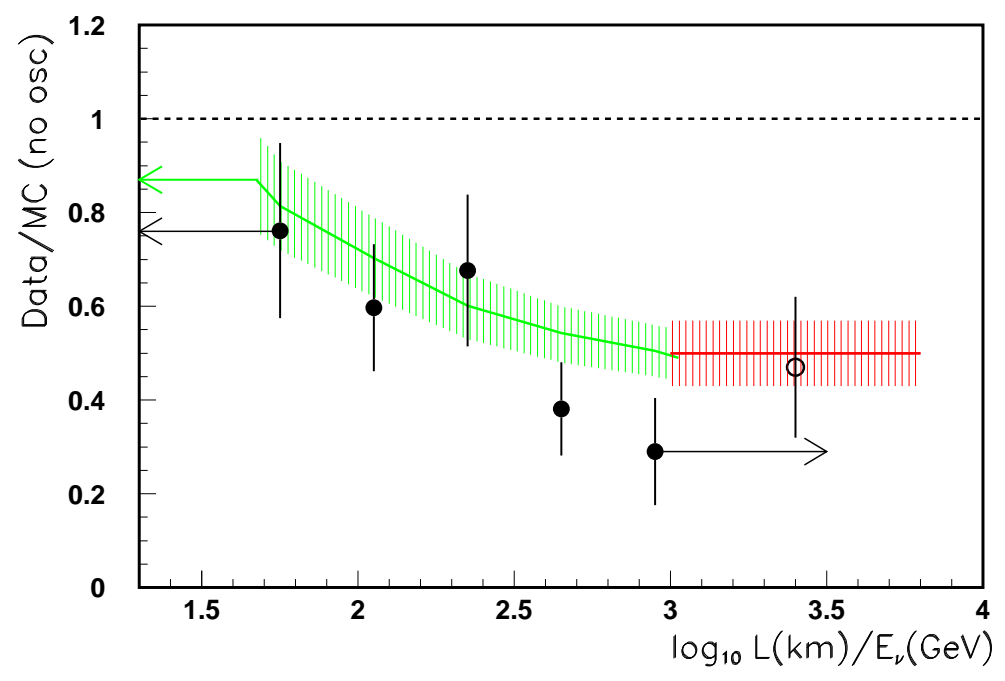

Figure 4: Ratio (Data/MC Bartol96) as a function of estimated $L / E_{\nu}$ for the upthrougoing muon sample (black circles) and the semicontained up- $\mu$ (open circle). For upthroughgoing muons the muon energy was estimated by multiple Coulomb scattering and $E_{\nu}$ by MC methods. The shaded regions represent the uncertainties in the MC predictions assuming $\sin ^{2} 2 \theta=1$ and $\Delta m^{2}=0.0023 \mathrm{eV}^{2}$. The horizontal dashed line at Data/MC=1 is the expectation for no oscillations.

yielding upgoing muons stopping in the detector. The semicontained downgoing muons (ID) are due to $\nu_{\mu}$-induced downgoing muon tracks with vertex in the lower MACRO. The two types of events (262 compared to 354 expected by Bartol96 and 315 by FLUKA, Table 1) are identified by means of topological criteria; the lack of time information prevents to distinguish the two sub-samples. The upgoing $\nu_{\mu}$ 's should all have oscillated completely, while the downgoing $\nu_{\mu}$ do not. The zenith distribution shows, as expected, a uniform deficit of about $25 \%$ of the measured number of events with respect to the no-oscillation prediction, Fig. 2] [5], [].

\section{Determination of the oscillation parameters}

In the past, in order to determine the oscillation parameters, MACRO made fits to the shape of the upthroughgoing muon distribution and to their absolute flux compared to the Bartol96 MC

\begin{tabular}{cccc}
\hline Ratio & $R_{\text {meas }} \pm \sigma_{\text {stat }}$ & $R_{0}$ & $R_{0} \leq R_{\text {meas }}$ probab \\
& & & a priori II \\
\hline Vertical/Horizontal & $1.38 \pm 0.12$ & 2.11 & $6.4 \times 10^{-6}$ \\
$N_{\text {low }} / N_{\text {high }}$ & $0.85 \pm 0.16$ & 1.5 & $7.7 \times 10^{-3}$ \\
InUp $/($ InDown + UpStop $)$ & $0.60 \pm 0.06$ & 0.745 & $3.1 \times 10^{-2}$ \\
\hline Combination & & & $3.4 \times 10^{-7}$ \\
\hline
\end{tabular}

Table 2: Ratios between different event categories: $R_{\text {meas }}$ is the measured value, $R_{0}$ is the expected value from the Bartol96 MC without oscillations; using the new FLUKA MC the ratios differ by at most $5 \%$. The last column gives the one-sided probability compatible with a statistical fluctuation computed according to the a priori assumptions explained in the text. 


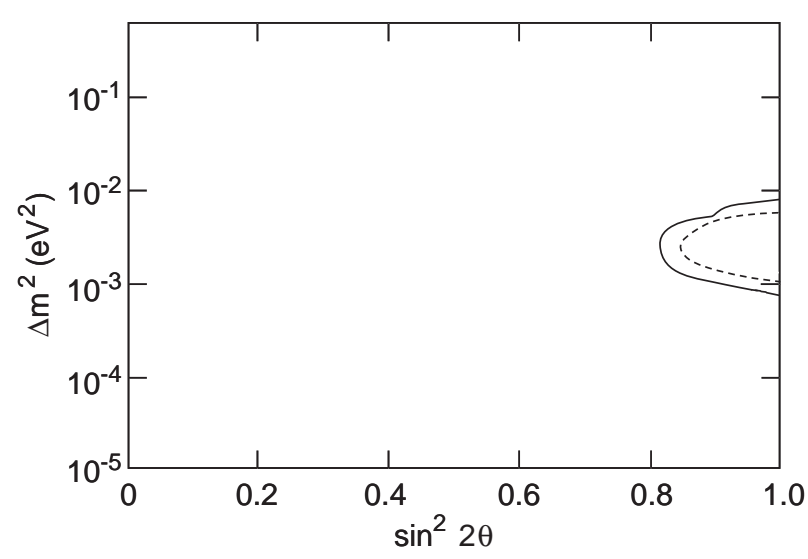

(a)

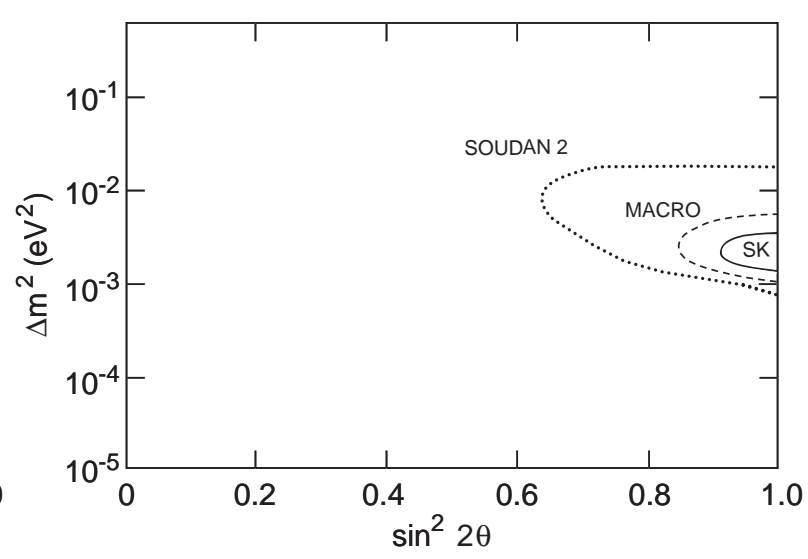

(b)

Figure 5: Interpolated qualitative $90 \%$ C.L. contour plots of the allowed regions in the $\Delta m^{2}-\sin ^{2} 2 \theta$ plane (a) for the MACRO data using only the ratios $R_{1}, R_{2}, R_{3}$ (outer continuous line) and using also the absolute values assuming the validity of the Bartol96 fluxes. (b) Comparison of the allowed regions for SK, MACRO and Soudan 2 (All contours have been smoothed out and must be considered as qualitative).

prediction (with a $17 \%$ systematic scale uncertainty). The other data were only used to verify the consistency and make checks. The result was $\Delta m^{2}=0.0025 \mathrm{eV}^{2}$ and maximal mixing.

Later, in [6, 7] the high energy data for the zenith distribution, $R_{1}=\left(N_{\text {vert }} / N_{\text {horiz }}\right)$, were combined with the HE neutrino energy measurement, $R_{2}=N_{\text {low }} / N_{\text {high }}$. They are independent of the neutrino absolute flux; with these two informations the significance of observation of neutrino oscillations is about $4.7 \sigma$.

In order to reduce the effects of possible uncertainties in the MCs (to about $6 \%$ ) we now use the following three independent ratios [8].

(i) High Energy Data: zenith distribution ratio: $R_{1}=N_{\text {vert }} / N_{\text {hor }}$

(ii) High Energy Data, neutrino energy measurement ratio: $R_{2}=N_{\text {low }} / N_{\text {high }}$

(iii) Low Energy Data: Ratio $R_{3}=(\text { Data } / M C)_{I U} /(\text { Data } / M C)_{I D+U G S}$.

Table 2 gives for each ratio the measured value, $R_{\text {meas }}$, the prediction, $R_{0}$, for no oscillations using either the Bartol96 or the new FLUKA MC; the probability value for no oscillations obtained allowing the predicted ratio to fluctuate around the mean value (considering the total number of events equal to the predicted value without oscillations). The no oscillations hypothesis has a probability $\mathrm{P} \sim 3.4 \times 10^{-7}$ (Table 2), ruling out the nooscillation hypothesis by $\sim 5 \sigma$. The formula used for combining independent probabilities is $P=P_{1} P_{2} P_{3}\left(1-\ln P_{1} P_{2} P_{3}+1 / 2\left(\ln P_{1} P_{2} P_{3}\right)^{2}\right)[20$.

By fitting the three ratios to the $\nu_{\mu} \rightarrow \nu_{\tau}$ oscillation formulae we obtain $\sin ^{2} 2 \vartheta=1, \Delta m^{2}=$ $2.3 \cdot 10^{-3} \mathrm{eV}^{2}$ and the allowed region indicated in Fig. $5 \mathrm{a}$.

We could also add the information on the absolute flux values of the

(iv) high energy data, assuming the validity of the Bartol96 flux and a systematic scale error of $17 \%, R_{4}=N_{\text {meas }} / N_{\text {Bartol-nooscill }} \simeq 0.73, P_{4} \simeq 0.07$

(v) low energy semicontained muons, with a systematic scale error of $21 \%$,

$R_{5}=N_{\text {meas }} / N_{\text {Bartol-nooscill }} \simeq 0.67, P_{5} \simeq 0.08$ (At these low energies the Bartol96, Honda 2001 and Fluka $2002 \mathrm{MC}$ neutrino fluxes are essentially equal). 
Fig. [5 shows the MACRO allowed regions for the $\nu_{\mu} \rightarrow \nu_{\tau}$ oscillation parameters in the $\Delta m^{2}-\sin ^{2} 2 \theta$ plane using only the three ratios $R_{1}, R_{2}$ and $R_{3}$ (continuous line) and using also the two estimated absolute values, assuming the validity of the Bartol96 fluxes (dashed line). In Fig. 5b are compared the MACRO, SuperKamiokande (SK) [16] and Soudan 2 [21] allowed regions. All limiting lines are qualitative smoothed interpolations.

\section{Neutrino Astrophysics with MACRO}

Search for Astrophysical Sources of High Energy Muon Neutrinos. High energy $\nu_{\mu}$ 's are expected to come from several galactic and extragalactic sources. Neutrino production requires astrophysical accelerators of protons and astrophysical "beam dumps". A sensitive search was made for upgoing muons produced by neutrinos coming from celestial sources, interacting below the detector. $90 \%$ c.l. upper limits were established on the muon fluxes from specific celestial sources; the limits are in the range $10^{-15}-10^{-14} \mathrm{~cm}^{-2} \mathrm{~s}^{-1}$. In the case of GX339-4 $\left(\alpha=255.71^{\circ}, \delta=-48.79^{\circ}\right)$ and $\operatorname{Cir} \mathrm{X}-1\left(\alpha=230.17^{\circ}, \delta=-57.17^{\circ}\right)$, we have 7 events: they have been considered as background, therefore the upper flux limits are higher; but the events could also be indication of signals [22, 2, 3. The pointing capability of the detector was tested by determining the shadows of the Moon and of the Sun on the primary cosmic ray flux [23]

A search for time coincidences of the upgoing muons with $\gamma$-ray bursts was made. No statistically significant time correlation was found. A search was also made for a diffuse astrophysical neutrino flux for which an upper limit at the level of $1.5 \cdot 10^{-14} \mathrm{~cm}^{-2} \mathrm{~s}^{-1}$ was established [24].

Indirect Searches for WIMPs.Weakly Interacting Massive Particles (WIMPs) could be part of the galactic dark matter; they could be intercepted by celestial bodies, slowed down and trapped in their centers, where WIMPs and anti-WIMPs could annihilate and yield neutrinos of multi GeV energy, in small angular windows from their centers. One of the best WIMP candidate could be the lowest mass neutralino.

A search was made for $\nu_{\mu} \rightarrow \mu$ coming from the center of the Earth using $10^{\circ}-15^{\circ}$ cones around the vertical; we obtained muon flux limits of $\sim 10^{-14} \mathrm{~cm}^{-2} \mathrm{~s}^{-1}$. The limits were compared with the predictions of a supersymmetric model by Bottino et al.; our data eliminate a sizable range of parameters used in the model [25, 3. A similar procedure was used to search for muon neutrinos from the Sun: the upper limits are at the level of about $1.5 \cdot 10^{-14} \mathrm{~cm}^{-2} \mathrm{~s}^{-1}[25$.

Neutrinos from Stellar Gravitational Collapses. A stellar gravitational collapse of the core of a massive star is expected to yield a burst of all types of neutrinos and antineutrinos with energies of $5-30 \mathrm{MeV}$ in $\sim 10 \mathrm{~s}$. The $\bar{\nu}_{e}$ 's can be detected via $\bar{\nu}_{e}+p \rightarrow n+e^{+}$in the liquid scintillator. About $120 \bar{\nu}_{e}$ events could have been detected in our 580 t liquid scintillator for a stellar collapse at the center of our Galaxy. Two separate electronic systems were used. Both had an energy threshold of $\sim 7 \mathrm{MeV}$ and recorded pulse shape, charge and timing informations of the positron. Following a $>7 \mathrm{MeV}$ trigger, the first system PHRASE lowered its threshold to $\sim 1 \mathrm{MeV}$ for $800 \mu \mathrm{s}$ in order to detect (with $\mathrm{a} \simeq 25 \%$ efficiency) the $2.2 \mathrm{MeV} \gamma$ released in the capture reaction $n+p \rightarrow d+\gamma$ induced by the neutron produced in the primary process. A redundant supernova alarm system was in operation, alerting the physicists on shift; a procedure was defined to alert the various supernova observatories around the world [26]. MACRO was completed at the end of 1994; the first parts of the detector started operation in 1989. No stellar gravitational collapses in our Galaxy were observed from 1989 to 2000. 


\section{Conclusions}

The MACRO detector took data from 1989 till the end of year 2000. Important results were obtained in all the items listed in the proposal.

In particular for atmospheric neutrino oscillations we analyzed different event topologies, different energies, exploited muon Coulomb multiple scattering in the detector to measure muon energies and studied the effects of using different MCs. All data are in agreement with the hypothesis of $\nu_{\mu} \rightarrow \nu_{\tau}$ oscillations, with maximal mixing and $\Delta m_{23}^{2} \simeq 2.3 \cdot 10^{-3} \mathrm{eV}^{2}$, and rule out the no oscillation hypothesis by $\sim 5 \sigma$.

Studies were made on muon neutrino astronomy [22, 23], seasonal, solar and sidereal variations 2] 28, and on possible Dark Matter candidates : WIMPs (looking for muon neutrinos from the centers of the Earth and of the Sun), Nuclearites and Q-balls [2], 27]. In the search for GUT Magnetic Monopoles MACRO obtained the best existing direct flux upper limit $\left(\Phi<1.4 \times 10^{-16} \mathrm{~cm}^{-2} \mathrm{~s}^{-1} \mathrm{sr}^{-1}\right)$ over the widest $\beta$ range $\left(4 \times 10^{-5}<\beta<1\right)[19$.

\section{Acknowledgments}

We would like to acknowledge the cooperation of all the members of the MACRO collaboration. MACRO was a collaboration of US and Italian Institutions plus one Moroccan group; see Ref. 1] for the list of Authors and Institutions.

\section{References}

[1] S. Ahlen et al., Nucl. Instr. Meth. Phys. Res. A324(1993)337.

M. Ambrosio et al., Nucl. Instr. Meth. Phys. Res A486(2002)663.

[2] G. Giacomelli et al., Lectures at the 6th Constantine Summer School, Algeria (2002), hep-ex/0211035.

[3] M. Ambrosio et al., Astrop. Phys. 9(1998)105; "LNGS Status report 2001", hep-ex/0206027.

[4] S. Ahlen et al., Phys. Lett. B357(1995)481.

[5] P. Bernardini, Vulcano Workshop, 1998, hep-ex/9809003

M. Ambrosio et al., Phys. Lett. B434(1998)451; Phys. Lett. B478(2000)5.

G. Giacomelli et al., Trieste ICTP School, hep-ph/9901355, hep-ex/0201032.

[6] M. Ambrosio et al., Phys. Lett. B517(2001)59; hep-ex/0106049.

[7] M. Ambrosio et al., physics/0203018, Nucl. Instr. Meth. Phys. Res A492(2002)376.

M. Ambrosio et al., hep-ex/0304037, Phys. Lett. B566(2003)35.

[8] M. Ambrosio et al., Eur. Phys. J. C36(2004)357.

G. Giacomelli and A. Margiotta, hep-ex/0406037.

[9] V. Agrawal et al., Phys. Rev. D53(1996)1314.

[10] M. Gluck et al., Z. Phys. C67(1995)433.

[11] P. Lipari et al., Phys. Rev. Lett. 74(1995)384. 
[12] W. Lohmann et al., "Energy loss of muons in the range 1 - 10000 GeV", CERN 85-03.

[13] http://wwwasd.web.cern.ch/wwwasd/geant/

[14] M. Honda et al., Phys. Rev. D64(2001)053011; Phys. Rev. D52(1995)4995.

[15] G. Battistoni et al., Astrop. Phys. 19(2003)269; "Erratum", Astrop. Phys. 19(2003)291. G. Battistoni et al., "High energy extension of the FLUKA atmospheric neutrino flux", HE2.4, 1399, ICRC 2003, Tsukuba, Japan.

[16] Y. Hayato, "Status of the Super-K, the K2K-2 experiments and JHF nu project", HEP EPS Conference, Aachen, 17-23 July 2003.

Y. Fukuda et al., Phys. Rev. Lett. 81(1998)1562; Phys. Lett. B433(1998)9.

[17] M. F. Crouch, Proceedings 20 th ICRC, Moscow, USSR 6(1987)165.

M. Aglietta et al., Astrop. Phys. 3(1995)311.

D. De Muth et al., hep-ex/0304016, (2003).

[18] R. Battiston, Rapporteur talk on "Direct measurements and origin of Cosmic Rays", ICRC 2003, Tsukuba, Japan.

T. Montaruli, Rapporteur talk on "High energy phenomena", ICRC 2003, Tsukuba, Japan.

[19] M. Ambrosio et al., hep-ex/0207020, Eur. Phys. J. C25(2002)511.

G. Giacomelli and L. Patrizii, hep-ex/0302011.

[20] B. Roe, Probability and statistics in experimental physics, Springer (1992), pag. 128.

[21] W.W.M. Allison et al., Phys. Lett. B449 (1999) 137.

T. Mann et al., Nucl. Phys. B Proc. Suppl. 91 (2001) 134; Nucl. Phys. B Proc. Suppl. 110 (2002)349.

[22] M. Ambrosio et al., Astrophys. J. 546(2001)1038; astro-ph/0002492,

[23] M. Ambrosio et al., Phys. Rev. D59(1999)012003; hep-ex/9807006, astro-ph/0302586.

[24] M. Ambrosio et al., astro-ph/0203181, Astrop. Phys. 19(2003)1.

S. Ahlen et al., Astrophys. J. 412(1993)301.

[25] M. Ambrosio et al., Phys. Rev. D60(1999)082002, hep-ex/9812020.

[26] S. Ahlen et al., Astrop. Phys. 1(1992)11.

M. Ambrosio et al., Astrop. Phys. 8(1998)123; paper submitted to Eur. Phys. J. C.

[27] E. Witten, Phys. Rev. D30(1984)272.

A. De Rujula and S.L. Glashow, Nature 312(1984)734.

D. Bakari et al, hep-ex/0004019 M. Ambrosio et al., Eur. Phys. J. C13(2000)453.

[28] M. Ambrosio et al., astro-ph/0211119, Phys. Rev. D 67, 042002(2003). 